\title{
A Highly Accurate Approximation of Conic Sections by Quartic Bézier Curves
}

\author{
Zhi Liu ${ }^{1,}$, Na Wei ${ }^{1}$, Jieqing Tan ${ }^{1}$, Xiaoyan Liu ${ }^{2}$ \\ ${ }^{1}$ School of Mathematics, Hefei University of Technology, Hefei, China \\ ${ }^{2}$ Department of Mathematics, University of La Verne, La Verne, USA
}

Email address:

liuzhi314@126.com (Zhi Liu), 458183314@qq.com (Na Wei), jieqingtan@hfut.edu.cn (Jieqing Tan), xliu@laverne.edu (Xiaoyan Liu)

To cite this article:

Zhi Liu, Na Wei, Jieqing Tan, Xiaoyan Liu. A Highly Accurate Approximation of Conic Sections by Quartic Bézier Curves. Applied and Computational Mathematics. Vol. 5, No. 2, 2016, pp. 40-45. doi: 10.11648/j.acm.20160502.11

\begin{abstract}
A new approximation method for conic section by quartic Bézier curves is proposed. This method is based on the quartic Bézier approximation of circular arcs. We give the upper bound of Hausdorff distance between the conic section and the quartic Bézier curve, and also show that the approximation order is eight. And we prove that our approximation method has a smaller upper bound than previous quartic Bézier approximation methods. A quartic $\mathrm{G}^{2}$-continuous spline approximation of conic sections is obtained by using the subdivision scheme at the shoulder point of the conic section.
\end{abstract}

Keywords: Conic Section, Quartic Bézier Curve, Hausdorff Distance, Approximation, $G^{2}$-Continuous, Subdivision Scheme

\section{Introduction}

It is well-known that besides the straight line, the conic sections are the simplest geometric entity. Conic sections are widely used in the fields of CAGD or CAD/CAM. Since the most of conic sections cannot be accurately represented by polynomials in explicit form, the parameter polynomials are used to approximate the conic sections. Bézier curves and surfaces [1-4] are the modeling tools widely used in $\mathrm{CAD} / \mathrm{CAM}$ systems. Most of the previous work on conic sections approximation is based on quartic Bézier curves.

In 1997, Ahn and Kim [5] presented the approximation of circular arcs by quartic and quintic Bézier curves with approximation orders eight and ten. The approximation of circular arcs by quartic Bézier curves with approximation order eight were represented in [6-8]. Fang [9] presented a method for approximating conic sections using quintic polynomial curves. The constructed quintic polynomial curve has $\mathrm{G}^{3}$-continuity with the conic section at the end points and $\mathrm{G}^{1}$-continuity at the parametric mid-point. Floater [10] found that the approximation of the conic section by Bézier curve of any odd degree $n$ has optimal approximation order $2 n$. Ahn [11] presented two methods of the quartic Bézier approximation of the conic section. $\mathrm{Hu}$ [12] gave a method for approximating conic sections by constrained Bézier curves of arbitrary degree. In 2014, $\mathrm{Hu}$ [13] provided a new approximation method of conic sections by quartic Bézier curves, which has a smaller error bound than previous quartic Bézier approximations.

The outline of this paper is as follows: In section 2, we present a new approximation method for conic sections by quartic Bézier curves, and give an upper bound on the Hausdorff distance between the conic section and the quartic Bézier curve. It is shown that the approximation order is eight. And we prove that our approximation method has a smaller error bound than previous quartic Bézier approximations. Finally, we illustrate our results by some numerical examples.

\section{Quartic Bézier Approximation of Conic Sections}

In this section, we give a new highly accurate approximation method of conic section by quartic Bézier curves. The conic section can be represented as [14]

$$
\boldsymbol{c}(\mathrm{t})=\frac{\mathrm{B}_{0}^{2}(\mathrm{t}) \boldsymbol{p}_{0}+\omega \mathrm{B}_{1}^{2}(\mathrm{t}) \boldsymbol{p}_{1}+\mathrm{B}_{2}^{2}(\mathrm{t}) \boldsymbol{p}_{2}}{\mathrm{~B}_{0}^{2}(\mathrm{t})+\omega \mathrm{B}_{1}^{2}(\mathrm{t})+\mathrm{B}_{2}^{2}(\mathrm{t})}
$$

where $\boldsymbol{p}_{0}, \boldsymbol{p}_{1}, \boldsymbol{p}_{2}$ are the control points, $\omega>0$ is the weight associated with $\boldsymbol{p}_{1}, \mathrm{~B}_{\mathrm{i}}^{2}(\mathrm{t})$ is the quadratic Bernstein polynomial given by 


$$
B_{i}^{2}(t)=\left(\begin{array}{l}
2 \\
i
\end{array}\right) t^{i}(1-t)^{2-i}, \quad i=0,1,2
$$

It is also well known that $c(t)$ is an ellipse when $\omega<1$, a parabola when $\omega=1$ and a hyperbola when $\omega>1$.

The quartic Bézier curve used to approximate the conic section $c(\mathrm{t})$ is given by

$$
\boldsymbol{b}(\mathrm{t})=\sum_{\mathrm{i}=0}^{4} \boldsymbol{b}_{\mathrm{i}} \mathrm{B}_{\mathrm{i}}^{4}(\mathrm{t})
$$

where $\boldsymbol{b}_{\mathrm{i}}(0 \leq \mathrm{i} \leq 4)$ are the control points, $B_{i}^{4}(t)(0 \leq i \leq 4)$ are the quartic Bernstein polynomials defined by

$$
B_{i}^{4}(t)=\left(\begin{array}{l}
4 \\
i
\end{array}\right) t^{i}(1-t)^{4-i}, \quad i=0,1,2,3,4
$$

Lemma 1. [15] Suppose that $\boldsymbol{h}(\mathrm{t}), \mathrm{t} \in[0,1]$, is any continuous curve which lies entirely inside the (closed) triangle $\Delta \boldsymbol{p}_{0} \boldsymbol{p}_{1} \boldsymbol{p}_{2}$ such that $\boldsymbol{h}(0)=\boldsymbol{p}_{0}$ and $\boldsymbol{h}(1)=\boldsymbol{p}_{2}$. Then

$$
\mathrm{d}_{\mathrm{H}}(\boldsymbol{h}, \boldsymbol{c}) \leq \frac{1}{4} \max \left(\frac{1}{\omega^{2}}, 1\right) \max _{\mathrm{t} \in[0,1]}|\mathrm{f}(\boldsymbol{h}(\mathrm{t}))|\left|\boldsymbol{p}_{0}-2 \boldsymbol{p}_{1}+\boldsymbol{p}_{2}\right|,
$$

where $d_{H}(\boldsymbol{h}, \boldsymbol{c})$ is the Hausdorff distance [12] defined by

$$
\mathrm{d}_{\mathrm{H}}(\boldsymbol{h}, \boldsymbol{c})=\max \left\{\max _{\mathrm{t} \in[0,1] \in[0,0,1]}|\boldsymbol{h}(\mathrm{t})-\boldsymbol{c}(\mathrm{s})|, \max _{\mathrm{s} \in[0,1] \in[0[0,1]} \min _{\mid}|\boldsymbol{h}(\mathrm{t})-\boldsymbol{c}(\mathrm{s})|\right\},
$$

and $f: \Delta \boldsymbol{p}_{0} \boldsymbol{p}_{1} \boldsymbol{p}_{2} \rightarrow \mathrm{R}$ is a function defined by

$$
\mathrm{f}(\mathrm{x})=\tau_{1}^{2}-4 \omega^{2} \tau_{0} \tau_{2},
$$

where $\tau_{0}, \tau_{1}, \tau_{2}$ are the barycentric coordinates with respect to $\Delta \boldsymbol{p}_{0} \boldsymbol{p}_{1} \boldsymbol{p}_{2}$. Any point $\mathrm{x} \in \Delta \boldsymbol{p}_{0} \boldsymbol{p}_{1} \boldsymbol{p}_{2}$ can be expressed as $\mathrm{x}=\tau_{0} \boldsymbol{p}_{0}+\tau_{1} \boldsymbol{p}_{1}+\tau_{2} \boldsymbol{p}_{2}$. The curve $\boldsymbol{c}(\mathrm{t})$ satisfies the equation $\mathrm{f}(\boldsymbol{c}(\mathrm{t}))=0$ for $\mathrm{t} \in[0,1]$.

The control points of the approximation curve $\boldsymbol{b}(\mathrm{t})$ can be expressed as

$$
\begin{gathered}
\boldsymbol{b}_{0}=\boldsymbol{p}_{0}, \quad \boldsymbol{b}_{1}=(1-\alpha) \boldsymbol{p}_{0}+\alpha \boldsymbol{p}_{1}, \quad \boldsymbol{b}_{2}=(1-\beta) \boldsymbol{m}+\beta \boldsymbol{p}_{1}, \\
\boldsymbol{b}_{3}=(1-\alpha) \boldsymbol{p}_{2}+\alpha \boldsymbol{p}_{1}, \boldsymbol{b}_{4}=\boldsymbol{p}_{2},
\end{gathered}
$$

where $\boldsymbol{m}=\frac{\boldsymbol{p}_{0}+\boldsymbol{p}_{2}}{2}$ is the midpoint of $\boldsymbol{p}_{0}$ and $\boldsymbol{p}_{2}$. In order to ensure that the approximation curves $\boldsymbol{b}(\mathrm{t})$ is contained in $\Delta \boldsymbol{p}_{0} \boldsymbol{p}_{1} \boldsymbol{p}_{2}, \alpha$ and $\beta$ must satisfy $0<\alpha<1$ and $0<\beta<1$.

The point $\boldsymbol{b}\left(\frac{1}{2}\right)$ lies on the line segment joining two points $\boldsymbol{p}_{1}$ and $m$, and $\boldsymbol{b}(\mathrm{t})$ has the barycentric coordinates with respect to $\Delta \boldsymbol{p}_{0} \boldsymbol{p}_{1} \boldsymbol{p}_{2}$

$$
\left\{\begin{array}{l}
\tau_{0}=(1-t)^{2}\left[(1-t)^{2}+4(1-\alpha) t(1-t)+3(1-\beta) t^{2}\right] \\
\tau_{1}=2 t(1-t)\left[2 \alpha(1-t)^{2}+3 \beta t(1-t)+2 \alpha t^{2}\right] \\
\tau_{2}=t^{2}\left[3(1-\beta)(1-t)^{2}+4(1-\alpha) t(1-t)+t^{2}\right] .
\end{array}\right.
$$

Obviously

$$
\tau_{\mathrm{i}}(\mathrm{i}=0,1,2)
$$

satisfy

$\tau_{0}(t)=\tau_{2}(1-t), \tau_{1}(t)=\tau_{1}(1-t)$. Substituting Eq.(3) into Eq.(2), we can get

$$
f(b(t))=-4 t^{2}(1-t)^{2}(A+B+C),
$$

where

$A=\left(\omega^{2}-1\right)(4 \alpha-3 \beta)^{2}\left(t-\frac{1}{2}\right)^{4}$,

$B=\left(\frac{9}{2} \beta^{2}\left(1-\omega^{2}\right)-8\left(1+\omega^{2}\right) \alpha^{2}+16 \omega^{2} \alpha-4 \omega^{2}\right)\left(t-\frac{1}{2}\right)^{2}$,

$\mathrm{C}=\frac{1}{16}[8 \omega-(4 \alpha+4 \beta)(1+\omega)][8 \omega+(4 \alpha+3 \beta)(1-\omega)]$.

Suppose $f(b(t))$ has zeros at $t=\frac{1}{2}$. Then from $\mathrm{f}\left(\boldsymbol{b}\left(\frac{1}{2}\right)\right)=0 \quad$ it $\quad$ follows $\quad \alpha=\frac{2 \omega}{\omega \pm 1}-\frac{3}{4} \beta \quad$. But $\alpha=\frac{2 \omega}{\omega-1}-\frac{3}{4} \beta$ will tend to infinity as $\omega$ tends to 1 , which does not meet our requirement. So we choose

$$
\alpha=\frac{2 \omega}{\omega+1}-\frac{3}{4} \beta .
$$

Substituting Eq.(5) into Eq.(4), we can get

$$
\mathrm{f}(\boldsymbol{b}(\mathrm{t}))=\frac{\mathrm{q}_{\beta}(\mathrm{t})}{(\omega+1)^{2}} 4 \mathrm{t}^{2}(\mathrm{t}-1)^{2}\left(\mathrm{t}-\frac{1}{2}\right)^{2},
$$

where

$$
\begin{gathered}
\mathrm{q}_{\beta}(\mathrm{t})=4\left(\omega^{2}-1\right)[3(\omega+1) \beta-4 \omega]^{2} \mathrm{t}(1-\mathrm{t})+9(\omega+1)^{2} \beta^{2} \\
+12 \omega(\omega+1)\left(\omega^{2}+\omega-4\right) \beta-4 \omega^{2}\left(3 \omega^{2}+6 \omega-13\right)
\end{gathered}
$$

The approximation curve $\boldsymbol{b}(\mathrm{t})$ contacts with the conic $c(t)$ at $\mathrm{t}=0, \frac{1}{2}$ and 1 with multiplicity at least two respectively. If $\mathrm{t}=\frac{1}{5}$ and $\mathrm{t}=\frac{4}{5}$ are the roots of $\mathrm{f}(\boldsymbol{b}(\mathrm{t}))=0$, we can get 


$$
\beta_{\mathrm{i}}=\frac{2 \omega}{3(\omega+1)} \cdot \frac{7 \omega^{2}-25 \omega+68-(-1)^{\mathrm{i}} 5(\omega-1) \sqrt{(\omega+1)(9 \omega+91)}}{16 \omega^{2}+9}, \mathrm{i}=1,2
$$

By Eqs. (6), (7) and (8) we can get the leading coefficient $\mathrm{u}_{\mathrm{i}}(\omega)$ of $\mathrm{f}(\boldsymbol{b}(\mathrm{t}))$ as follows

$$
u_{i}(\omega)=2^{6} 5^{2} \frac{\omega^{2}(1-\omega)^{3}\left[5(\omega+2)+(-1)^{i} \sqrt{(9 \omega+91)(\omega+1)}\right]^{2}}{(\omega+1)\left(16 \omega^{2}+9\right)^{2}}, i=1,2 .
$$

Since the approximation curve $\boldsymbol{b}(\mathrm{t})$ is chosen to contact with the conic $c(t)$ at $t=0, \frac{1}{5}, \frac{1}{2}, \frac{4}{5}, 1$ with multiplicity 2,1,2,1 and 2, we have

$$
\mathrm{f}\left(\boldsymbol{b}_{\mathrm{i}}(\mathrm{t})\right)=\mathrm{u}_{\mathrm{i}}(\omega) \mathrm{t}^{2}\left(\mathrm{t}-\frac{1}{5}\right)\left(\mathrm{t}-\frac{1}{2}\right)^{2}\left(\mathrm{t}-\frac{4}{5}\right)(\mathrm{t}-1)^{2} .
$$

From $\left|\mathrm{f}\left(\boldsymbol{b}_{2}(\mathrm{t})\right)\right|>\left|\mathrm{f}\left(\boldsymbol{b}_{1}(\mathrm{t})\right)\right|$, it follows

$$
|\mathrm{f}(\boldsymbol{b}(\mathrm{t}))|=\left|\mathrm{f}\left(\boldsymbol{b}_{1}(\mathrm{t})\right)\right|=\left|\mathrm{u}_{1}(\omega)\right| \mathrm{t}^{2}\left(1-\frac{1}{2}\right)^{2}(\mathrm{t}-1)^{2}\left|\left(\mathrm{t}-\frac{1}{5}\right)\left(\mathrm{t}-\frac{4}{5}\right)\right| \text {. }
$$

The value of $|f(\boldsymbol{b}(\mathrm{t}))|$ is only determined by $\boldsymbol{\omega}$. If we want to determine an upper bound on the Hausdorff distance between the approximation curves and the conics, we need to obtain the range of $\omega$ to ensure that the approximation curve $\boldsymbol{b}(\mathrm{t})$ lies inside $\Delta \boldsymbol{p}_{0} \boldsymbol{p}_{1} \boldsymbol{p}_{2}$.

Theorem 1. If the weight $\omega$ satisfies $0<\omega<\omega_{1}^{\prime}$, then the curve $\boldsymbol{b}_{1}(\mathrm{t})$ lies inside $\Delta \boldsymbol{p}_{0} \boldsymbol{p}_{1} \boldsymbol{p}_{2}$, where $\alpha\left(\omega_{1}^{\prime}\right)=1$ and $\omega_{1}^{\prime} \approx 5.8331$.

Proof. According to the convex hull property of Bézier curves, the quartic Bézier curve $\boldsymbol{b}_{1}(\mathrm{t})$ lies inside $\Delta \boldsymbol{p}_{0} \boldsymbol{p}_{1} \boldsymbol{p}_{2}$ when $0<\alpha<1$ and $0<\beta_{1}<1$.

Substituting $\beta=\beta_{1}$ into Eq. (5), we can get

$$
\alpha=\frac{\omega[(\omega+1)(57 \omega-32)-5(\omega-1) \sqrt{(9 \omega+91)(\omega+1)}]}{2(\omega+1)\left(16 \omega^{2}+9\right)} \text {. }
$$

Differentiating $\alpha$ with respect to $\omega$ gives

$$
\frac{\mathrm{d} \alpha}{\mathrm{d} \omega}=\frac{256 \omega^{2}+513 \omega-144}{\left(16 \omega^{2}+9\right)^{2}}+\frac{2560 \omega^{4}-12090 \omega^{3}-14030 \omega^{2}-5535 \omega+4095}{2(\omega+1)\left(16 \omega^{2}+9\right)^{2} \sqrt{(9 \omega+91)(\omega+1)}}
$$

Since the equation $\frac{d \alpha}{d \omega}=0$ has no real roots, there are only two possibilities, either $\frac{\mathrm{d} \alpha}{\mathrm{d} \omega}>0$, or $\frac{\mathrm{d} \alpha}{\mathrm{d} \omega}<0$ for all $0<\omega<\infty$.

From $\lim _{\omega \rightarrow 0} \frac{\mathrm{d} \alpha}{\mathrm{d} \omega} \approx 0.8721>0$, it follows $\frac{\mathrm{d} \alpha}{\mathrm{d} \omega}>0$ for all $0<\omega<\infty$. Therefore $\alpha$ is a monotonically increasing function with respect to $\omega$ for $0<\omega<\infty$. It is easy to get $\lim _{\omega \rightarrow 0} \alpha=0, \lim _{\omega \rightarrow \infty} \alpha \approx 1.3125>1$. Let $\alpha\left(\omega_{1}^{\prime}\right)=1$. Then

$$
\omega_{1}^{\prime}=\frac{(14615-6 \mathrm{i} \sqrt{5006517})^{\frac{1}{3}}}{15}+\frac{(14615+6 \mathrm{i} \sqrt{5006517})^{\frac{1}{3}}}{15}+\frac{7}{3} \approx 5.8331 .
$$

Similarly, differentiating $\beta_{1}$ with respect to $\omega$ gives

$$
\begin{gathered}
\frac{\mathrm{d} \beta_{1}}{\mathrm{~d} \omega}=\frac{2}{3} \cdot \frac{512 \omega^{4}-2050 \omega^{3}-1124 \omega^{2}-450 \omega+612}{(\omega+1)^{2}\left(16 \omega^{2}+9\right)^{2}} \\
+\frac{2}{3} \cdot \frac{\sqrt{\omega+1}\left(-2560 \omega^{4}+12090 \omega^{3}+14030 \omega^{2}+5535 \omega-4095\right)}{\sqrt{9 \omega+91}(\omega+1)^{2}\left(16 \omega^{2}+9\right)^{2}} .
\end{gathered}
$$

The equation $\frac{\mathrm{d} \beta_{1}}{\mathrm{~d} \omega}=0$ has a unique zero $\omega_{2}^{\prime} \approx 9.9106$. Since $\beta_{1}\left(\omega_{2}^{\prime}\right) \approx 0.9514, \quad \lim _{\omega \rightarrow 0} \beta_{1}=0, \lim _{\omega \rightarrow \infty} \beta_{1} \approx 0.9167$, we have $0<\beta_{1}<\beta_{1}\left(\omega_{2}^{\prime}\right)<1$ for any $\omega \in(0,+\infty)$.

In summary, we have $0<\alpha<1,0<\beta_{1}<1$ for $0<\omega<\omega_{1}^{\prime}$.

Theorem 2. For $0<\omega<\omega_{1}^{\prime}$, the Hausdorff distance between the conic section $\boldsymbol{c}(\mathrm{t})$ and the approximation curve $\boldsymbol{b}_{1}(\mathrm{t})$ is bounded as

$$
\mathrm{d}_{\mathrm{H}}\left(\boldsymbol{b}_{1}, \boldsymbol{c}\right) \leq \delta(\omega)\left|\boldsymbol{p}_{0}-2 \boldsymbol{p}_{1}+\boldsymbol{p}_{2}\right|
$$

where

$\delta(\omega)=\frac{50053+95489 \sqrt{2329}}{2^{13} 5^{6}} \max \left(\frac{1}{\omega^{2}}, 1\right) \frac{\omega^{2}|1-\omega|^{3}[5(\omega+2)-\sqrt{(9 \omega+91)(\omega+1)}]^{2}}{(\omega+1)\left(16 \omega^{2}+9\right)^{2}}$

Proof. The polynomial $\mathrm{t}^{2}\left(1-\frac{1}{2}\right)^{2}(\mathrm{t}-1)^{2}\left|\left(\mathrm{t}-\frac{1}{5}\right)\left(\mathrm{t}-\frac{4}{5}\right)\right|$ has the maximum $\frac{50053+95489 \sqrt{2329}}{2^{17} 5^{8}}$ at $\mathrm{t}=\frac{1}{2} \pm \frac{\sqrt{17}+\sqrt{137}}{40}$ in the interval $[0,1]$. By Eq.(1) and Eq.(9), we can get the value of $\delta(\omega)$. The proof of Theorem 2 is completed.

Floater [15] gave the result that $\omega-1$ and $\left|\boldsymbol{p}_{0}-2 \boldsymbol{p}_{1}+\boldsymbol{p}_{2}\right|$ are $\mathrm{o}\left(\mathrm{h}^{2}\right)$, where $\mathrm{h}$ is the maximum length of the parametric interval under subdivision. So according to the error bound, the approximation order of the approximation curve $\boldsymbol{b}_{1}(\mathrm{t})$ in Theorem 2 is eight. The error of Hu's approximation method [13] is smaller than that of other previous quartic Bézier curve approximation methods. Next, we will prove our error bound is smaller than that of Hu's method.

Hu showed in [13] that for $0<\omega<\omega_{2} \approx 5.753038$ 


$$
\mathrm{d}_{\mathrm{H}}\left(\boldsymbol{Q}_{2}, \boldsymbol{p}\right) \leq \lambda(\omega)\left|\boldsymbol{p}_{0}-2 \boldsymbol{p}_{1}+\boldsymbol{p}_{2}\right|
$$

where

$$
\lambda(\omega)=\frac{3(471+133 \sqrt{57})}{2^{19}} \max \left(\frac{1}{\omega^{2}}, 1\right) \frac{\omega^{2}|\omega-1|^{3}[2(\omega+2)-\sqrt{(\omega+1)(\omega+15)}]^{2}}{(\omega+1)\left(3 \omega^{2}+1\right)^{2}} .
$$

Theorem 3. For $0<\omega<\min \left(\omega_{2}, \omega_{1}^{\prime}\right)=\omega_{2} \approx 5.753038$, the upper bound on the Hausdorff error (10) by our method is smaller than that by Hu's method, i.e., $\delta(\omega)<\lambda(\omega)$.

Proof. Comparing $\delta(\omega)$ in (10) with $\lambda(\omega)$ in (11) reveals that to show $\delta(\omega)<\lambda(\omega)$ is equivalent to proving the following inequality

$$
\eta(\omega)=\frac{5(\omega+2)+\sqrt{(9 \omega+91)(\omega+1)}}{2(\omega+2)+\sqrt{(\omega+1)(\omega+15)}}>\sqrt{\frac{64(50053+95489 \sqrt{2329})}{5^{6} \times 3(471+133 \sqrt{57})}} \approx 2.0764 .
$$

It is obvious that $\frac{\mathrm{d} \eta}{\mathrm{d} \omega}>0$ for $0<\omega<\min \left(\omega_{2}, \omega_{1}^{\prime}\right)=\omega_{2}$ according to Fig 1. Therefore

$$
\eta(\omega)>\eta(0) \approx 2.4818>2.0764
$$

as asserted.

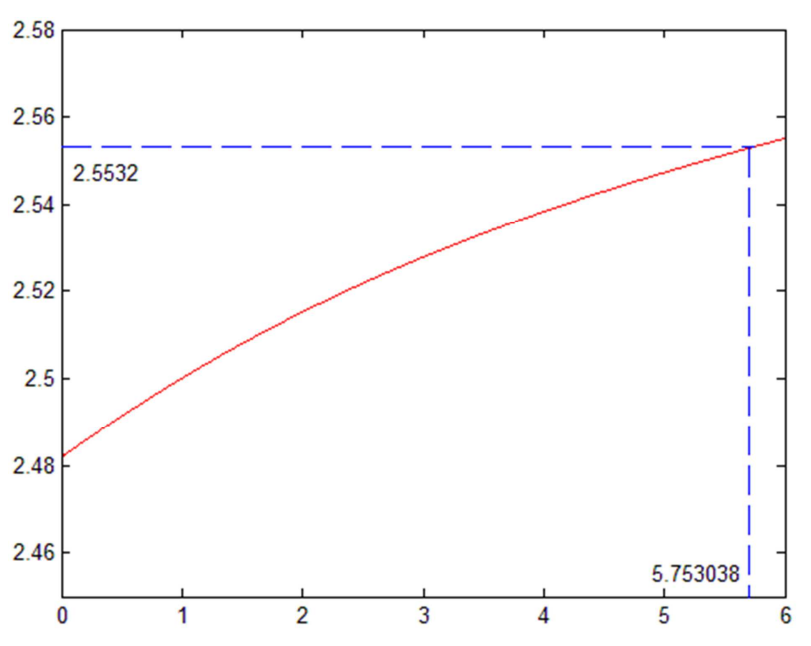

Fig. 1. The graph of $\eta(\omega)$.

\section{Numerical Examples}

Example 1. Let the conic section $c(\mathrm{t})$ be given with the control points $\boldsymbol{p}_{0}=(0,0), \boldsymbol{p}_{1}=(120,150), \quad \boldsymbol{p}_{2}=(100,0)$ and the weight $\omega=0.5$, as shown in Fig 2(a). The quartic Bézier $\boldsymbol{b}_{1}(\mathrm{t})$ has the control points $\boldsymbol{b}_{0}=\boldsymbol{p}_{0}=(0,0)$, $\boldsymbol{b}_{1}=(37.9560,47.4450) \quad, \quad \boldsymbol{b}_{2}=(82.6970,70.0650) \quad$, $\boldsymbol{b}_{3}=(106.3260,47.4450), \boldsymbol{b}_{4}=\boldsymbol{p}_{2}=(100,0)$, as shown in Fig 2(b). The Hausdorff error bound is

$$
\mathrm{d}_{\mathrm{H}}\left(\boldsymbol{b}_{1}, \boldsymbol{c}\right) \leq 1.7 \times 10^{-3}
$$

by Theorem 2 in our method. Obviously, this error bound is smaller than that with $\mathrm{Hu}$ 's method, which is

$$
\mathrm{d}_{\mathrm{H}}\left(\mathrm{Q}_{2}, \boldsymbol{p}\right) \leq 2.413908 \times 10^{-3}
$$

obtained by (11).

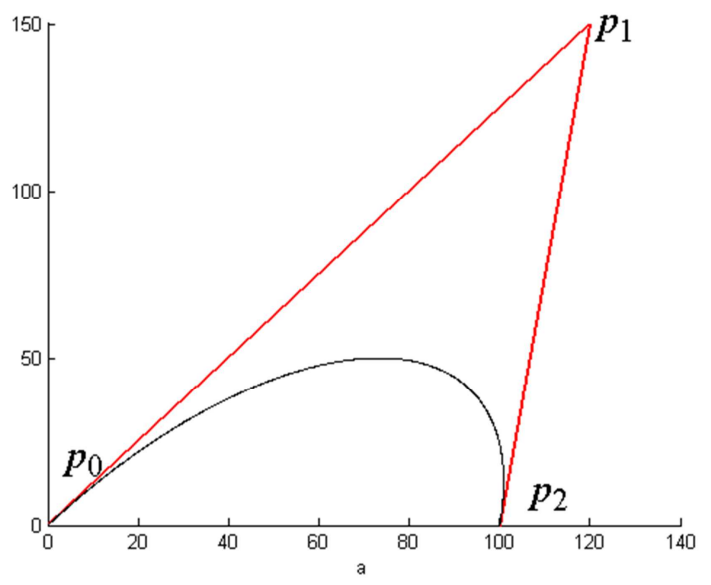

(a) the conic section $c(\mathrm{t})$

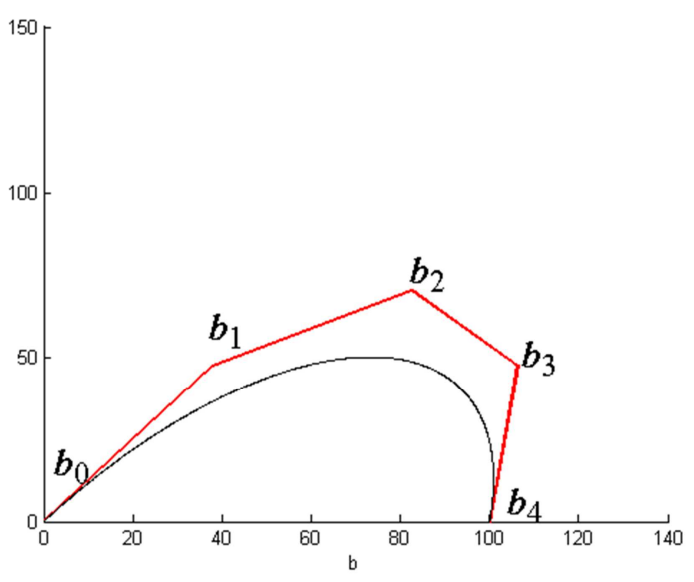

(b) the quartic Bézier approximation $\boldsymbol{b}_{1}(\mathrm{t})$

Fig. 2. The quartic Bézier approximation of conic section with $\omega=0.5$.

Example 2. Let the conic section $c(\mathrm{t})$ be given with the control points $\boldsymbol{p}_{0}=(0,0), \quad \boldsymbol{p}_{1}=(120,150), \quad \boldsymbol{p}_{2}=(100,0)$ and the weight $\omega=3$, as shown in Fig 3(a). The quartic Bézier $\boldsymbol{b}_{1}(\mathrm{t})$ has the control points $\boldsymbol{b}_{0}=\boldsymbol{p}_{0}=(0,0)$, $\boldsymbol{b}_{1}=(99.6360,124.5450) \quad, \quad \boldsymbol{b}_{2}=(112.5100,133.9500)$, $\boldsymbol{b}_{3}=(116.6060,124.5450), \quad \boldsymbol{b}_{4}=\boldsymbol{p}_{2}=(100,0)$, as shown in Fig. 3(b). The Hausdorff error bound is

$$
\mathrm{d}_{\mathrm{H}}\left(\boldsymbol{b}_{1}, \boldsymbol{c}\right) \leq 0.993 \times 10^{-1}
$$

by Theorem 2 in our method. Obviously, this error bound is smaller than that with Hu's method, which is

$$
\mathrm{d}_{\mathrm{H}}\left(\mathrm{Q}_{2}, \boldsymbol{p}\right) \leq 1.471996 \times 10^{-1}
$$

obtained by (11). 


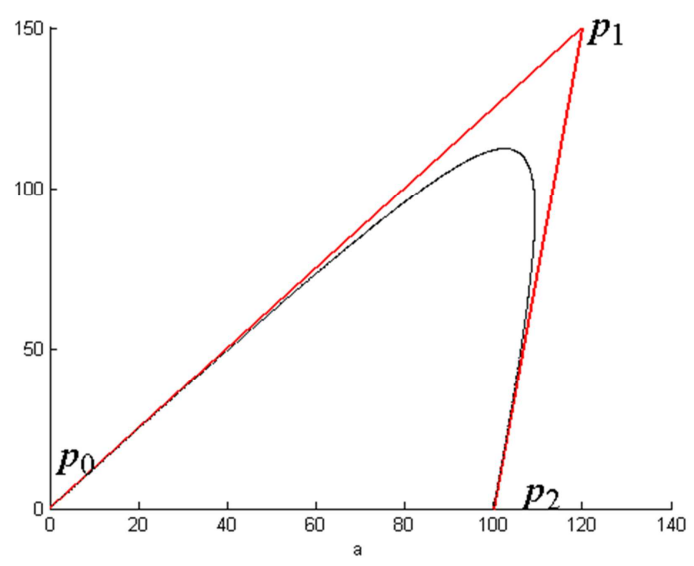

(a) the conic section $c(\mathrm{t})$;

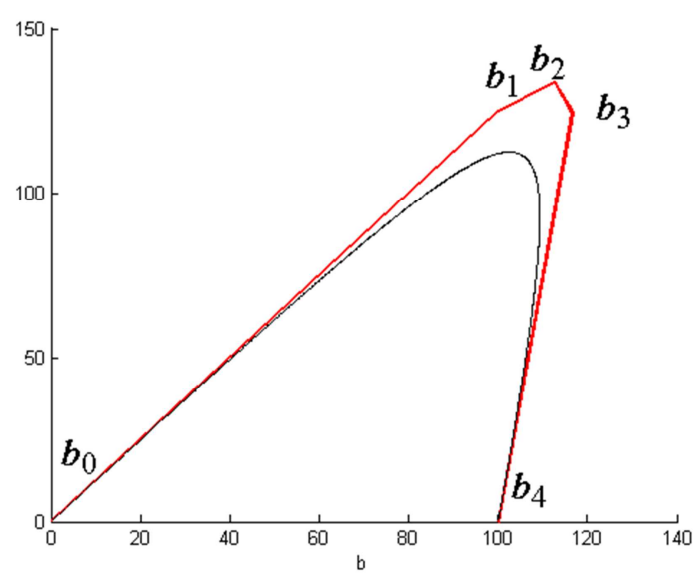

(b) the quartic Bézier approximation $\boldsymbol{b}_{1}(\mathrm{t})$

Fig. 3. The quartic Bézier approximation of conic section with $\omega=3$.

In addition, if the bound on the Hausdorff error $d_{H}\left(b_{1}, c\right)$ is larger than a user-specified error tolerance, we can consider the subdivision scheme for $c(t)$, consisting of alternately subdividing at the shoulder point $\boldsymbol{c}(0.5)$ and normalizing each subcurve, as stated in [15]. Using this subdivision scheme, the composite curve of the quartic Bézier approximation curve $\boldsymbol{b}_{1}(\mathrm{t})$ is globally $G^{2}$ continuous. Suppose the conic section $c(\mathrm{t})$ is subdivided at $\boldsymbol{c}(0.5)$ into two parts $c_{1}(\mathrm{t})$ and $c_{2}(\mathrm{t})$. Then $c_{1}(\mathrm{t})$ and $c_{2}(\mathrm{t})$ have control points as

$$
p_{0}^{1}=p_{0}, \quad p_{1}^{1}=\frac{p_{0}+\omega p_{1}}{1+\omega}, \quad p_{2}^{1}=\frac{p_{0}+2 \omega p_{1}+p_{2}}{2(1+\omega)}=p_{0}^{2}, \quad p_{1}^{2}=\frac{p_{2}+\omega p_{1}}{1+\omega}, \quad p_{2}^{2}=p_{2}
$$

and the weight $\omega=\sqrt{\frac{\omega+1}{2}}$ associated with the control points $\boldsymbol{p}_{1}^{1}$ and $\boldsymbol{p}_{1}^{2}$.

In Example 2, If the error tolerance is $\varepsilon=0.001$, then we should split the conic $c(t)$ at $c(0.5)$ into two segments $c_{1}(\mathrm{t})$ and $\boldsymbol{c}_{2}(\mathrm{t})$, as shown in Fig 4(a), at the shoulder point by the subdivision scheme proposed in [16].

Using our method, the quartic Bézier approximations $\boldsymbol{b}_{1}^{1}(\mathrm{t})$ and $\boldsymbol{b}_{1}^{2}(\mathrm{t})$ have the Hausdorff error bounds

$$
\mathrm{d}_{\mathrm{H}}\left(\boldsymbol{b}_{1}^{1}, \boldsymbol{c}_{1}\right) \leq 2.7049 \times 10^{-4}, \quad \mathrm{~d}_{\mathrm{H}}\left(\boldsymbol{b}_{1}^{2}, \boldsymbol{c}_{2}\right) \leq 2.2931 \times 10^{-4} .
$$

The composition curve of $\boldsymbol{b}_{1}^{1}(\mathrm{t})$ and $\boldsymbol{b}_{1}^{2}(\mathrm{t})$ yields the quartic $G^{2}$ continuous spline approximation $\boldsymbol{b}_{1}(\mathrm{t})$ of the conic section $c(\mathrm{t})$, as shown in Fig. 4(b).

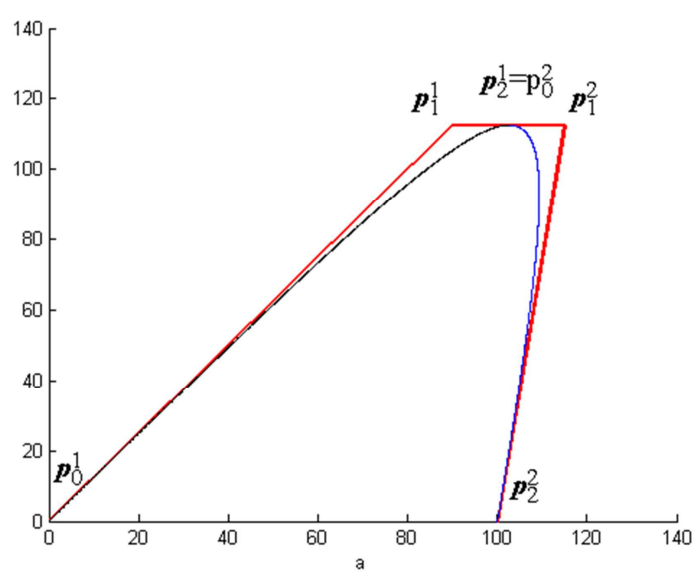

(a)The conic section $c_{1}(\mathrm{t})$ and $\boldsymbol{c}_{2}(\mathrm{t})$.

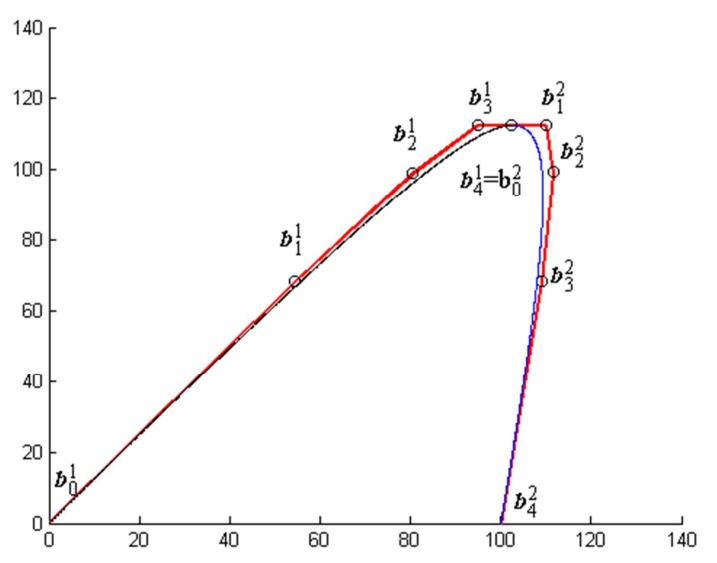

(b) The composite curve of $\boldsymbol{b}_{1}^{1}(\mathrm{t})$ and $\boldsymbol{b}_{1}^{2}(\mathrm{t})$.

Fig. 4. The $G^{2}$ continuous quartic Bézier approximation curve.

\section{Conclusion}

We give a new approximation method for conic section by quartic Bézier curves, and prove that our approximation method has a smaller error bound than previous quartic Bézier approximations. Although the approximations are not optimal, but the result is high accuracy. The next question considered is to find a better zeros sequence in order to have smaller error bound.

\section{Acknowledgements}

The authors would like to thank the referees for their valuable comments which greatly help improve the clarity and quality of the paper.

This work was supported in part by the National Natural 
Science Foundation of China (Grant No. 61472466 and 11471093), Key Project of Scientific Research, Education Department of Anhui Province of China under Grant No. KJ2014ZD30. The Fundamental Research Funds for the Central Universities under Grant No. JZ2015HGXJ0175.

\section{References}

[1] Li-Wen Han, Ying Chu, Zhi-Yu Qiu, Generalized Bézier curves and surfaces based on Lupaş q-analogue of Bernstein operator. Journal of Computational and Applied Mathematics 261(2014) 352-363.

[2] J. Sánchez-Reyes. The conditions for the coincidence or overlapping of two Bézier curves. Applied Mathematics and Computation 248(2014) 625-630.

[3] Przemysław Gospodarczyk, Degree reduction of Bézier curves with restricted control points area. Computer-Aided Design 62 (2015) 143-151.

[4] Çetin Dişibüyük, Ron Goldman, A unifying structure for polar forms and for Bernstein Bézier curves. Journal of Approximation Theory 192(2015) 234-249.

[5] Young Joon Ahn, Hong Oh Kim. Approximation of circular arcs by Bézier curves, Journal of Computational and Applied Mathematics 81(1997): 145-163.

[6] Seon-Hong Kim, Young Joon Ahn. An approximation of circular arcs by quartic Bézier curves, Computer-Aided Design 39(2007) 490-493.
[7] Zhi Liu, Jie-qing Tan, Xiao-yan Chen, Li Zhang. An approximation method to circular arcs, Applied Mathematics and Computation 219(2012) 1306-1311.

[8] Boštjan Kovač, Emil Žagar. Some new $G^{1}$ quartic parametric approximants of circular arcs. Applied Mathematics and Computation 239(2014) 254-264.

[9] Lian Fang. $G^{3}$ approximation of conic sections by quintic polynomial curves, Computer Aided Geometric Design 16(1999) 755-766.

[10] Michael S. Floater, An o $\left(\mathrm{h}^{2 \mathrm{n}}\right)$ Hermite approximation for conic sections, Computer Aided Geometric Design 14(1997) $135-151$.

[11] Young Joon Ahn, Approximation of conic sections by curvature continuous quartic Bézier curves, Computers \& Mathematics with Applications 60(2010) 1986-1993.

[12] Qian-qian $\mathrm{Hu}$, Approximating conic sections by constrained Bézier curves of arbitrary degree, Journal of Computational and Applied Mathematics 236(2012) 2813-2821.

[13] Qian-qian $\mathrm{Hu}, G^{1}$ approximation of conic sections by quartic Bézier curves, Computers \& Mathematics with Applications 68(2014) 1882-1891.

[14] Lian Fang, A rational quartic Bézier representation for conics, Computer Aided Geometric Design 19(2002): 297-312.

[15] Michael S. Floater. High order approximation of conic sections by quadratic splines, Computer Aided Geometric Design 12(1995) 617-637.

[16] Young Joon Ahn, Conic approximation of planar curves, Computer-Aided Design 33(2001): 867-872. 